\title{
Development of management tools in the context of NBIC convergence
}

\author{
Antonina Pahomova ${ }^{1}$, Sándor Halász ${ }^{2}$, Galina Zelenkova, ${ }^{3, *}$ and Alexander Pakhomov ${ }^{4}$ \\ ${ }^{1}$ Platov South-Russian State Polytechnic University (NPI), Department of Production and \\ Innovation Management, Novocherkassk, Russia \\ ${ }^{2}$ Budapest University of Technology and Economics, Department of Electric Power \\ Engineering, Budapest, Hungary \\ ${ }^{3}$ Don State Technical University, sq. Gagarina, 1, Rostov-on-Don, 344003, Russia \\ ${ }^{4}$ Don State Agrarian University, o. Persianovsky, 346493, Russia
}

\begin{abstract}
The greatest success in improving the breeding and productive qualities of Hereford cattle can be achieved through the use of high-value breeding bulls. The role of producers at the modern stages of beef cattle breeding has increased dramatically, so the evaluation of bulls by the quality of offspring has become an important breeding event to improve and create highly productive herds of beef cattle. At the same time, the use of bulls that are predisposed to various diseases or the deterioration of their offspring can cause irreparable negative consequences for beef cattle breeding. In the course of research work in the conditions of the farm, studies were conducted to assess the quality of bulls-producers of the Hereford breed of cattle. Evaluation of Hereford bulls by the quality of offspring in farm conditions will increase the efficiency of herd reproduction technology in beef cattle breeding, allow rational use of bulls, get a high yield of calves (90-95\%), organize the accuracy of accounting for the origin of young animals, increase the level of breeding work to improve the productive, breeding qualities of animals and create highly productive herds of Hereford cattle in a shorter time. Keywords: beef cattle breeding, Hereford breed, breeding bulls, bull evaluation, quality of offspring, creation of highly productive herds.
\end{abstract}

\section{Introduction}

The global crisis cannot be resolved according to the previous fundamental patterns in the paradigm of the development of our civilization. Modern technologies require colossal amounts of energy, which the existing alternative energy is not able to generate. We need a qualitative leap, a transition to other principles of energy production and consumption based on the model of living nature, which change the face of the entire technosphere,

\footnotetext{
* Corresponding author: zelenkovalex@rambler.ru
} 
namely, nature-like convergent NBIC technologies that provide natural resource circulation (Kovalchuk, 2011; Kovalchuk, Naraikin \& Yatsishina, 2019). However, at the same time, such technologies generate fundamentally new global threats (Kovalchuk \& Naraikin, 2016):

- the dual nature of technologies, blurred boundaries between civil and military use and, as a result, the inefficiency of existing means and technologies of control;

- impossibility to predict all the consequences of the release of artificial living systems into the environment;

- biogenetic nanobiotechnologies, allowing the production of artificial living systems with desired properties, on the basis of which both super-effective drugs and means of destruction can be created;

- cognitive technologies based on the convergence of infocognitive and sociohumanitarian sciences and affecting the psychophysiological sphere of a person in order to control and manage his mind and body.

NBIC convergence in production systems requires new approaches to HR resource management. The cost of error for each decision in such systems is several orders of magnitude higher than in traditional systems (Som \& Hilty, 2007; Royakkers, Timmer, Kool \& Van Est, 2018; Angelopoulou, Mykoniatis \& Boyapati, 2020), and can lead to other errors which, spreading exponentially, can lead to a global world catastrophe. It is obvious that the human factor is decisive here (Kolbachev \& Kolbacheva, 2019).

Technological evolution contributes to an increase in structural and dynamic complexity caused by the heterogeneity and unanticipated behavior of system components. In this regard, human-machine interaction and human-robot interaction can be considered as sources of risk. These risks are associated with new technological and organizational variables, an increase in the number of risk sources, and an increase in the likelihood of exposure to external influences. The emerging risks are developing towards scenarios with greater uncertainty due to the great flexibility of cyber-physical systems, an increase in the complexity of the tasks performed, a combination of human error and artificial intelligence error. The reliability of such devices is more difficult to predict as the complexity of these systems increases. Human monitoring of processes is performed through systems control instead of direct control, which can lead to a reduction in practical knowledge of the process and an over-reliance on automated safety systems. Psychosocial and musculoskeletal risks are associated with low physical activity, high mental stress, decreased confidentiality at work, and increased decision-making problems. (Brocal, González, Komljenovic, Katina \& Sebastián, 2019)

It is necessary to find not only tools to control, optimize and develop human production activities, but also mechanisms for the formation and evolution of cultural and worldviews and, as a consequence, new meanings and concepts that allow for sustainable development of mankind.

According to (Robert, Giuliani \& Gurau, 2020), the Industry 4.0 paradigm proposes a new form of manufacturing organization based on direct control of the entire value chain and implemented through the Internet of Things IoT, BigData and cloud technologies integrated into cyber-physical production. In managing new systems, the problem of human constraints must be addressed.

BigData technology allows you to collect, analyze and improve not only production processes, but also human behavior. At the same time, Industry 4.0 performs selfoptimization in real time, whereas previously this required forecasts. Working conditions are changing, a new working environment is emerging. Man already performs not just the function of managing production systems, but is a teacher for artificial intelligence of machines. All human actions are recorded and analyzed, forming a behavioral memory (knowledge base). 
To solve this problem, it is necessary to develop a methodology that would allow the development of an effective and safe human-centered production system in the context of NBIC convergence.

\section{Methods}

The proposed methodology is based on the systemic paradigm of J. Kornai (Kornai, 2002), developed in subsequent studies (Kleiner, 2002) - the concept of seeing the object and the subject of research, according to which the socio-economic space is considered as a single system that contains many relatively independent subsystems, the composition and structure of which is determined in accordance with the position of the observer or a group of observers. The reasons for the emergence and spread of the systemic paradigm were associated with the crisis of orthodox economic theory. At the same time, the socioeconomic system is understood as a relatively isolated and stable part or a certain aspect of the socio-economic space-time continuum, which is characterized by external integrity and internal diversity. Within the framework of this paradigm, any technical system (which is essentially socio-technical, because it provides for human participation in its creation and use) can be viewed as just such a subsystem located in a certain socio-economic space. The systemic paradigm of J. Kornai was used as the basis for the understanding of the production system as a multi-dimensional system that combines heterogeneous elements and resources (Kolbachev, 2003).

As the studied elements of the system, it is proposed to consider human health control in eHealth, production safety control, human capital management through HR analytics, human-machine interaction and the cognitive decision-making system.

Health is a fundamental element in the development and effective performance of a person, which is the ultimate goal of any economic, technological and social development, namely, the provision of permanent health (Maksimović, Vujović, \& Perišić, 2016).

EHealth Networked eHealth includes continuous prevention, proactive monitoring, follow-up, and real-time management of chronic illness by healthcare professionals using portable IoMT devices that provide patient health data. The devices are equipped with the following types of sensors:

- sensors of inertial motion;

- bioelectric sensors;

- electrochemical sensors;

- optical sensors;

- temperature sensors.

Sensors collect information about health determinants such as:

- position and movement of the body;

- changes in temperature, blood pressure and heart rate;

- glucose content, oxygen level and concentration of carbon dioxide in the blood;

- the nature of respiration, galvanic skin response and muscle activity.

The system takes into account not only the internal state of a person, but also the characteristics of the social, economic, cultural and environment in which he is. Install devices that assess climatic conditions, the chemical composition of the air, radiation, noise and vibration levels, temperature, humidity, lighting and other parameters of the workspace, continuously monitoring compliance with the standards.

Thus, human health is considered in interaction with the environment, one of the key factors of which is work safety. The system, by means of sensors, allows identifying cases of a person being in the danger zone and / or signaling operations that may lead to an accident.

Here, issues related to ergonomics, workspace optimization, control of physical, informational and psychological stress can also be resolved. 
The next step in the development of this technology is the introduction of nanomedicine through IoNT, which allows you to control the functioning of all human organs.

The use of HR analytics based on the data accumulated through BigData technology and predictive analytics implemented using artificial intelligence, machine learning, modeling, optimization methods and heuristics allows solving the following tasks (Nocker \& Sena, 2019):

- human capital management (measuring the performance of personnel, determining the optimal configuration of the team in terms of a set of skills and knowledge);

- human resources analytics (identifying the driving forces of productivity and their contribution to overall productivity);

- forecasting alternative personnel scenarios;

- strategy for building human resources;

- personnel recruiting;

- professional development and development (an individual training plan for employees based on their education and nature of work; determining the benefits of investment in training);

- productivity and compensation payments (building a performance management system for employees based on their individual achievements and compensation payments for certain results);

- employee involvement and motivation (development of motivational programs to support personnel involvement, determination of the driving forces of staff turnover);

- network analysis (identification and improvement of connections between employees and / or teams in organizations);

- analysis and forecasting of employee sentiment to identify potential problems.

In the time management of employees' work, time should be divided into two categories: subjective and objective. Objective time is chronological time, which is the same for all. Subjective time is a relativistic time that depends on many different factors: a person's ability to control time, feelings, a person's cultural background, situation, time pressure, lack of sleep, personal qualities or personal plans, etc. These factors affect a person's perception of time and divert it from objective time. (Reunanen, 2015)

Analytics for solving issues of optimization of time management in production systems are often based on surveys, which are usually conducted after the completion of tasks, depend on the mental state and character of the employee, and therefore cannot be completely reliable. Analytics in production systems of the fifth and sixth technological modes is formed in real time on the basis of objective information about the time and quality of a particular job.

Machine learning is an artificial intelligence technology that allows machines to learn and then make decisions without human intervention by learning patterns, creating logical and functional connections from multidimensional data, operational and behavioral predictions. Types of machine learning (Sassi, Anter \& Bekkhoucha, 2019):

- supervised learning: all data is tagged and algorithms are trained to predict the output from the input data based on data classification according to the teacher's requirements;

- unsupervised learning: data is not tagged, and algorithms are trained based on internal structures consisting of input data;

- learning with partial involvement of the teacher: some data are labeled, but most of them are not labeled (used when it is necessary to understand the structure of the data and perform classification in order to be able to predict);

- reinforcement learning: the algorithm learns behavior from observation.

Machine learning techniques (Sassi, Anter \& Bekkhoucha, 2019):

- classification: a forecasting method that assigns database objects (situations) to one of the predefined classes according to a predefined classification model; 
- regression: a certain target variable is predicted from a known dataset of the observed object;

- clustering: identification and creation of homogeneous subsets (clusters) to describe data from a heterogeneous common set of objects in the database;

- dimensionality reduction: data transformation is aimed at using the internal data structure, which consists in reducing the number of variables by obtaining the main variables;

- teaching associative rules: establishing associative rules based on the discovery of relationships between variables in the database;

- Anomaly detection: This is the prediction and identification of elements or events that do not match the expected pattern or other elements present in the dataset.

In this case, through double iterative connections, a person acts as a teacher, and a machine, learning, corrects a person's actions.

To manage risks associated with the human factor, it is advisable to use the experience of nuclear power, on the basis of which the need for the following measures was formulated (Brocal, González, Komljenovic, Katina \& Sebastián, 2019):

- regular discussion of risks, emerging difficulties and their interrelationships;

- analysis of discrepancies between forecasts and the real situation;

- lobbying for a different opinion in order to avoid willful carelessness (reduction of cognitive and motivational biases);

- analysis and discussion of previous work;

- doubts and uncertainties should not remain unanswered;

- demonstration of system security;

- extending defense in depth to fully cover complex systems and their intrinsic nonlinearity.

Decision making should be complex and multi-level, the implementation and security of which is possible using blockchain technology.

Blockchain technology distributes decision making among all parties that cannot be distorted or reversed due to two properties (Tapus \& Manolache, 2019):

- Transparency: data is distributed among all nodes of the network, everyone has access to the entire history of transactions or decisions that were made up to a certain point. Any new decisions or transactions will automatically take into account the previous decisions.

- Incorruptible: the data stored on the blockchain cannot be changed, as locally changed units of information in any given block will create a mismatch between the changed blockchain with all other nodes in the network.

So, in the work (Tapus \& Manolache, 2019), an integrated environment for decentralized decision-making is proposed, implemented through a blockchain in the image of the distribution of data between cells and their storage within DNA, modeled on the basis of human anatomy and various natural phenomena and processes, such as the decision-making process human neurons, the nature of the organization of natural fractals, bee swarms and ant colonies.

On the basis of the presented methods, a person-oriented concept of HR-resources management of the production system in the context of NBIC convergence was developed.

\section{Results}

In the developed human-oriented concept of HR-resources management of the production system in the context of NBIC-convergence, the main actors are "Man" and "Machine". Their interaction is due to double and global iterative bonds. Each person $\mathrm{H}$ corresponds to a machine $\mathrm{M}$ (or their combination). The person controls and trains the machine. The machine, in turn, accumulates the history of all operations $\mathrm{O}$ of a person, interacting in parallel with other machines and analyzing their efficiency based on a given target 
production function. Forming a knowledge base, as well as on the basis of existing models, the machine predicts and offers a person various rational scenarios of behavior. Thus, the risk of opportunistic behavior of a person is reduced: all his actions are supported by a multi-dimensional system analysis. The interaction "Human" - "Human" is necessary to improve production algorithms, introduce a greater share of the cognitive component into them and correct actions in teamwork.

For each task $\mathrm{j}$, a matrix of possible operations $\mathrm{O}$ is generated by the $\mathrm{i}$-th pair (or set) of a person $\mathrm{H}$ and a machine $\mathrm{M}$

$$
F_{i j}=\left[\begin{array}{cccc}
O_{11} & O_{12} & \ldots & O_{1 n} \\
O_{21} & O_{22} & \ldots & O_{2 n} \\
\ldots & \ldots & \ldots & \ldots \\
O_{m 1} & O_{m 2} & \ldots & O_{m n}
\end{array}\right],
$$

where $\mathrm{n}$ is the number of possible options for performing the operation, $\mathrm{m}$ is the number of operations.

Each operation $\mathrm{O}$ is represented by a set of values of $\mathrm{k}$ parameters $\mathrm{P}$ describing it

$$
O=\left\{P_{1}, P_{1}, \ldots, P_{k}\right\} \text {. }
$$

Taking into account the constraints, the value of the objective production function determined by means of the output parameters is compared with the set of matrices $\mathrm{F}$

$$
E=\left[\begin{array}{cccc}
\mathrm{F}_{11} & \mathrm{~F}_{12} & \ldots & \mathrm{F}_{1 \mathrm{j}} \\
\mathrm{F}_{21} & \mathrm{~F}_{22} & \ldots & \mathrm{F}_{2 \mathrm{j}} \\
\ldots & \ldots & \ldots & \ldots \\
\mathrm{F}_{\mathrm{i} 1} & \mathrm{~F}_{\mathrm{i} 2} & \ldots & \mathrm{F}_{\mathrm{ij}}
\end{array}\right] .
$$

Thus, the presented model makes it possible to predict the result of making certain production decisions or to propose rational options for scenarios for each pair of H-M that provide optimal values of $\mathrm{E}$ for given conditions.

Human capital management through HR analytics involves analyzing the current state and development of a person's competencies. The possession of competencies $\mathrm{C}$ is determined by the function of the time $t$ spent on performing the operation $\mathrm{O}$ and the level of achievement of the target production function $\mathrm{E}$

$$
C=f(E, t) \text {. }
$$

A time-based analysis of the implementation of competencies allows us to identify factors affecting the productivity of human resources, on the basis of which command configurations are optimal in terms of a set of skills and knowledge, time management tasks are solved, individual training plans for employees and a system for managing their performance are developed.

Safety sensors located in the workspace, functional sensors of machines and human physiological sensors allow timely detection of anomalies (emergency situations) and appropriate decisions.

Continuous monitoring of human health in the eHealth system ensures the stability of the system. Reducing the risks of unexpected permanent or temporary loss of employee performance eliminates the fact of unjustified investment in human resources.

Security is implemented through a decentralized decision-making system in the blockchain. At the same time, depending on the nature of the task, a combination of decision-making experts is automatically determined based on the rating system. An expert can be either a person $\mathrm{H}$ or a machine $\mathrm{M}$. The rating for each set of competencies is formed on the basis of the performance assessment by the pairs H-H, H-M, M-H, M-M. Man evaluates the performance of machines and other participants in the same way a machine evaluates the performance of humans and other machines. Thus, an integrated rating is formed for the sets of competencies for a person 
and machines

$$
R_{H}=f\left(R_{H}^{H}, R_{H}^{M}\right)
$$

$$
R_{M}=f\left(R_{M}^{H}, R_{M}^{M}\right)_{3}
$$

where $R_{H^{H}}^{H}$ - rating $\mathrm{H}$, formed by group $\mathrm{H} ; R_{H}^{M}$ - rating $\mathrm{H}$, formed by group $\mathrm{M} ; R_{M^{-}}^{H}$ rating $\mathrm{M}$, formed by group $\mathrm{H} ; R_{M}^{M}$ - rating $\mathrm{M}$, formed by group $\mathrm{M}$.

The proposed system guarantees objective collective decision-making by experts, the level of competence of which is determined by the history of their activities, analyzed both by artificial intelligence, which excludes the human factor, and by a person with cognitive perception.

\section{Discussion}

The presented human-oriented concept of HR-resources management in the context of NBIC-convergence has all the tools for iterative self-development and self-improvement, providing safe working conditions and constant monitoring of human health, which maximizes the percentage of return on human resources and reduces the level of opportunistic behavior.

This system is closed, and therefore has a certain limit in its development. To move to a new stage, a person needs to get out of the system to form new meanings and concepts, which is impossible without a humanitarian and cultural component, as well as without interaction between research and production clusters.

Humanity is approaching the moment of transition from a mechanistic paradigm to a holistic one, from an entropic stage to a syntropic one. At present, civilization is at an entropy stage, since it is primarily oriented towards profit. Transformation is associated with the human mind, and the mind is primarily associated with syntropy, the creation of a new paradigm and overcoming entropy, which confirms the need to form the correct value system. To achieve a unique spiral growth, it is necessary to break the resistance of the average value of the current balance of cognition and reach the critical (starting) point of spiral growth. (Mikulášková, Čambál, Polakovič \& Urbanovičová, 2020)

Thus, a spiral development model is proposed, each turn of which will be a selforganizing system.

\section{Conclusion}

The problem of control, optimization and evolution of the human factor in NBIC production systems is solved through the developed human-oriented concept of HRresources management, the development of which occurs in a spiral. Each turn of the spiral is a self-organizing system. For the current stage of development, a cyber-physical system of interaction "Man" - "Machine" is proposed, based on global and double iterative connections, parallel analysis, scenario forecasting, machine learning and the cognitive component. Human capital management is performed through HR analytics and BigData technology, predictive analytics implemented using artificial intelligence, machine learning, modeling, optimization methods and heuristics. Continuous monitoring of human health is implemented in the eHealth system, and continuous monitoring of production safety through sensors. Blockchain decision making is collective and decentralized.

This concept allows:

- control, optimize and develop human production activities;

- to provide iterative self-development and self-improvement of the system;

- guarantee the stability and safety of the system;

- maximize the percentage of return on human resources; 
- reduce the level of opportunistic human behavior.

The transition to a new stage of development is provided by the exit from the system for the formation of new meanings and concepts at the expense of the humanitarian and cultural component, as well as through interaction between research and production clusters.

\section{Acknowledgements}

The reported study was funded by RFBR and FRLC, project number 20-510-23002 "The development of Janos Kornai's system paradigm is the fundamental basis for the creation of production and technical systems in the conditions of NBIC convergence".

\section{References}

1. A. Angelopoulou, K. Mykoniatis, \& N.R. Boyapati, Procedia Manufacturing, 42, 296301. doi: 10.1016/j.promfg.2020.02.094 (2020)

2. F. Brocal, C. González, D. Komljenovic, P.F. Katina \& M.A. Sebastián, Complexity, 113. doi: $10.1155 / 2019 / 2089763$ (2019)

3. E. Kolbachev, T. Kolbacheva, AHFE 2018: Advances in Manufacturing, Production Management and Process Control, 793, 179-190. doi: 10.1007/978-3-319-94196-7_17 (2019)

4. M. Maksimović, V. Vujović \& B. Perišić, Journal of Information Systems Engineering \& Management, 1 (1), 25-39. doi: 10.20897/lectito.201607 (2016)

5. J. Mikulášková, M. Čambál, L. Polakovič \& P. Urbanovičová, Networked Business Models in the Circular Economy, 174-199. doi: 10.2507/29th.daaam.proceedings.131 (2020).

6. M. Nocker \& V. Sena, Social Sciences, 8(10), 273. doi: 10.3390/socsci8100273 (2019)

7. T. Reunanen, Procedia Manufacturing, 3, 709-716. doi: 10.1016/j.promfg.2015.07.311 (2015)

8. M. Robert, Ph. Giuliani. \& C. Gurau, Production Planning \& Control. doi: 10.1080/09537287.2020.1810761 (2020)

9. L. Royakkers, J. Timmer, L. Kool, L. \& R. Van Est, Ethics and Information Technology, 20, 127-142. doi: 10.1007/s10676-018-9452-x (2018)

10. I. Sassi, S. Anter \& A. Bekkhoucha, Advances in Intelligent Systems and Computing, 915, 237-251. doi: 10.1007/978-3-030-11928-7_21 (2019)

11. N. Tapus \& M.A. Manolache, Procedia Computer Science, 162, 587-595. doi: 10.1016/j.procs.2019.12.027 (2019)

12. M.V. Kovalchuk \& O.S. Naraikin, Safety index, 22 (3-4), 118-119 (2016)

13. M.V. Kovalchuk, Russian Nanotechnologies, 6 (13-23), 455-465. doi: 10.31857 / S0869-5873895455-465 (2011)

14. M.V. Kovalchuk, O.S. Naraikin \& E.B. Yatsishina, Bulletin of the Russian Academy of Sciences, 89 (5), 455-465. doi: 10.31857 / S0869-5873895455-465 (2019) 